\title{
There Is No Association of Radioactive Iodine Treatment with Cancer Mortality in Patients with Hyperthyroidism
}

\author{
Bennett S. Greenspan, Jeffry A. Siegel ${ }^{1}$, Aamna Hassan², and Edward B. Silberstein ${ }^{3}$ \\ ${ }^{1}$ Nuclear Physics Enterprises, Orlando, Florida; ${ }^{2}$ Shaukat Khanum Memorial Cancer Hospital and Research Center, Lahore, \\ Pakistan; and ${ }^{3}$ University of Cincinnati Medical Center, Cincinnati, Ohio
}

$\mathbf{T}$ he appropriate use of radioactive iodine therapy in hyperthyroidism has been undermined by the recent paper of Kitahara et al. in JAMA Internal Medicine, published online on July 1, 2019 (1). The paper is flawed in many ways, and we are writing to refute the claims made by Kitahara et al. This paper uses the same hyperthyroid patient cohort of the original paper by Saenger et al. in 1968 (2) and the follow-up paper by Ron et al. in 1998 (3). These 2 papers $(2,3)$ reviewed the use of ${ }^{131}$ I therapy (RAI [radioactive iodine]) for treatment of hyperthyroidism and compared RAI with treatment with antithyroid drugs (ATDs) and thyroid surgery. The current paper by Kitahara et al. follows this patient cohort for another $24 \mathrm{y}$ and investigates a dose-risk relationship for these hyperthyroid patients; however, the authors did not compare risk of cancer mortality with RAI with risk of ATD therapy or thyroid surgery. There was no appropriate control group, a basic methodologic error. Also, there was no correction for other more significant risk factors for cancer, especially smoking, which are known to have significant causal relationships with cancer risk, as acknowledged by the authors.

Saenger et al. and Ron et al. $(2,3)$ both concluded that RAI was a safe treatment for hyperthyroidism and neither found any increase in cancer mortality compared with ATDs and thyroid surgery. In the paper by Ron et al., there was a significant increase in cancer mortality from ATDs compared with RAI (3).

One of the most important issues, the known increase in cancer incidence and cancer mortality in patients with hyperthyroidism $(4,5)$, especially Graves' disease, was not considered in the Kitahara paper. Hyperthyroidism itself has a known positive association with an increased risk of thyroid and breast cancer (4); leukemia (2); and colon, lung, prostate, and breast malignancy (5). Saenger et al. reported that the risk of leukemia in hyperthyroid patients was $50 \%$ greater than in the U.S. population (2), yet Kitahara et al. calculated no dose-response relationship at all for leukemia in these patients. This is because they used assumptive model-based calculations that are likely incorrect and additionally because the causative factor of hyperthyroidism overwhelms the assumed low-dose contribution to the leukemia risk. Ryödi et al. (6) in a comparison of RAI therapy and thyroid surgery found that "the increased cancer risk in hyperthyroid patients is attributable to hyperthyroidism and shared risk factors, not the treatment modality."

Received Aug. 30, 2019; revision accepted Sep. 23, 2019.

For correspondence or reprints contact: Bennett S. Greenspan, 150 River Club Lane, North Augusta, SC 29841.

E-mail: bengreenspan0708@gmail.com

Published online Oct. 4, 2019.

COPYRIGHT @ 2019 by the Society of Nuclear Medicine and Molecular Imaging. DOI: 10.2967/jnumed.119.235929
Much more importantly, a very recent publication (7) by 2 of the coauthors of Kitahara et al. is now contradicting their findings: "The overall perception of death from cancer risk associated with ${ }^{131} \mathrm{I}$ is inflated and not supported by evidence. As co-authors of this article, we offer previously unpublished data and analysis that 1) disputes clinical significance of the associated risk from ${ }^{131} \mathrm{I}$ and 2) shows, again, that antithyroid drugs carry a statistically significant and a much more obvious cancer death risk." And "In conclusion, the Kitahara et al. publication provides a numeric estimate of excess cancer deaths after RAIT using assumptive model-based calculations. No excess cancer deaths were actually observed after ${ }^{131} \mathrm{I}$ treatment relative to that predicted in contemporaneous population (using SMR analyses). In contrast, ATD treatment is strongly associated with the excess cancer deaths in TTFUS [Cooperative Thyrotoxicosis Follow Up Study (Saenger (2)]. Due to the article's sole focus on the hypothetical and very doubtful ${ }^{131}$ I-associated cancer risks, it completely missed the highly statistically and clinically significant ATD-associated cancer risks that begs for immediate attention."

There is no effect at all below an exposure of $0.1 \mathrm{~Gy}(10 \mathrm{rad})$, as shown in their own graph (Figure B in Kitahara et al. (1)). Results above $0.1-0.5$ Gy (10-50 rad) show no dose response. It is reported that an exposure to the stomach of 0.17 Gy $(17 \pm 18$ rad) causes an increase in all solid cancer mortality, but that inexplicably exposure to the esophagus of $1.60 \mathrm{~Gy}(160 \pm 150 \mathrm{rad})$, 10 times as much, does not cause an increase in solid cancer mortality. Interestingly, there is no statistically significant increase in leukemia. The incidence of cancer mortality noted in the patients treated with RAI, $15.3 \%$, is actually substantially less than that in the general U.S. population of $20 \%-25 \%$ (8). In light of the known increased cancer mortality due to hyperthyroidism, this result of only $15.3 \%$ is notable in being so small. As stated by Giovanella and Verburg, "In fact, as in spite of nearly 8 decades of radioiodine therapy, no study has yet been able to prove the latter hypothesis" [a relationship between radioiodine therapy for hyperthyroidism and cancer mortality] $(9,10)$.

Dose modeling was used instead of actual dose assessment, and these dose estimates have very large error bars. Using these dose estimates to correlate with patient risk, estimated using an assumed, but not supported, linear model, is problematic. The linear nothreshold dose-risk model is simply assumed; this is known to be inaccurate (11) and is not even supported by the reported data. As mentioned above, the data shown in Figure B in this paper (1) do not indicate a linear dose-response relationship for breast cancer, even though this relationship is reported to be statistically significant. Thus, the authors have attempted to find a dose-risk 
relationship even though both the assumed doses and the risks are likely highly inaccurate. Therefore, the authors' assumption that association is related to causation and other conclusions have little basis in reality (12).

Most of the doses reported in this paper are very low and have not been previously shown to cause an increase in cancer mortality. The breast exposure of 15 rad would not be expected to cause an increase in cancer incidence or mortality.

The conclusion of the Kitahara paper, "In RAI-treated patients with hyperthyroidism, greater absorbed doses appeared to be modestly positively associated with risk of death from solid cancer, including breast cancer" is not justified by the data, as indicated by Tulchinsky and Brill (7). This lack of justification is further supported by the findings of a previous article by the Kitahara group analyzing a different cohort of individuals (13) as noted by Giovanella and Verburg (10), that indicate increased breast cancer mortality in women with hyperthyroidism over $60 \mathrm{y}$ of age, but no effect of RAI on this association.

In summary, major flaws of this paper include (1) poorly characterized dose estimates that were not directly measured and therefore have enormous and unknown uncertainties; (2) an analysis that does not even support the paper's conclusion that there is an association between RAI therapy and increased cancer mortality-there is no effect below $0.1 \mathrm{~Gy}$ (10 rad), and no dose response up to 0.5 Gy (50 rad); (3) the estimated doses are too low to expect increased cancer incidence or mortality; (4) since the relative risks are likely inaccurate, comparing them with the assumed and not directly measured doses is meaningless, especially since there are other known causative factors that are much more important contributors to cancer risk; (5) the known increase in cancer mortality associated with hyperthyroidism was not considered; (6) the greater cancer mortality of ATD therapy compared with ${ }^{131}$ I therapy was ignored; and (7) the assumption that a mild association indicates causation is unsupported.

The real harm of this paper is that clinicians may decline to use ${ }^{131}$ I therapy for the treatment of hyperthyroidism, in the belief that they are protecting their patients from cancer. In reality, aside from the fact that no evidence is presented of cancer risk at low doses, the use of ATDs will actually increase cancer incidence and mortality, in addition to the other problems with ATDs, including hepatic toxicity and agranulocytosis.
Based on the major flaws discussed above, and the major harm to clinical practice we anticipate, we suggest the readers submit a rebuttal paper to JAMA Internal Medicine.

\section{DISCLOSURE}

No potential conflict of interest relevant to this article was reported.

\section{REFERENCES}

1. Kitahara CM, Berrington de Gonzalez A, Bouville A, et al, Association of radioactive iodine treatment with cancer mortality in patients with hyperthyroidism. JAMA Intern Med. 2019;179:1034-1042.

2. Saenger EL, Thoma GE, Tompkins EA. Incidence of leukemia following treatment of hyperthyroidism: preliminary report of the Cooperative Thyrotoxicosis Therapy Follow-Up Study. JAMA. 1968;205:855-862.

3. Ron E, Doody MM, Becker AB, et al. Cancer mortality following treatment for adult hyperthyroidism. Cooperative Thyrotoxicosis Therapy Follow-up Study Group. JAMA. 1998;280:347-355.

4. Chen Y-K, Lin CL, Chang YJ, et al. cancer risk in patients with Graves' disease: a nationwide cohort study. Thyroid. 2013;23:879-884.

5. Moeller LC, Fuhrer D. Thyroid hormone, thyroid hormone receptors, and cancer: a clinical perspective. Endocr Relat Cancer. 2013;20:R19-R29.

6. Ryödi E, Metso S, Jaatinen P, et al. Cancer incidence and mortality in patients treated with RAI or thyroidectomy for hyperthyroidism. J Clin Endocrinol Metab. 2015;100:3710-3717.

7. Tulchinsky M, Brill AB. Spotlight on the association of radioactive iodine treatment with cancer mortality in patients with hyperthyroidism is keeping the highest risk from antithyroid drugs in the blind spot. Clin Nucl Med. 2019;44:789791.

8. National Research Council of the National Academies. Health Risks from Exposure to Low Levels of Ionizing Radiation: BEIR VII. Washington, DC: The National Academies Press; 2006.

9. Verburg FA, Luster M, Lassman M, Reiners C. ${ }^{131}$ I therapy in patients with benign thyroid disease does not conclusively lead to a higher risk of subsequent malignancies. Nucl Med (Stuttg). 2011;50:93-99.

10. Giovanella L, Verburg FA. Use of anti-thyroid drugs in patients with hyperthyroidism: a case for shared decision-making. Eur J Nucl Med Mol Imaging. August 9, 2019 [Epub ahead of print].

11. Siegel JA, Brooks AL, Fisher DR, et al. A critical assessment of the linear nothreshold hypothesis: its validity and applicability for use in risk assessment and radiation protection. Clin Nucl Med. 2019;44:521-525.

12. Grady E, Zukotynski K, Greenspan BS. Response to Kitahara. JAMA Intern Med. In press.

13. Journy NMY, Bernier MO, Doody MM, Alexander BH, Linet MS, Kitahara CM. Hyperthyroidism, hypothyroidism, and cause-specific mortality in a large cohort of women. Thyroid. 2017;27:1001-1010. 